\section{Mortalidad por neumonía y legionelosis en España: un estudio de series temporales}

\author{
ENRIQUE GEA-IZQUIERDO ${ }^{1, \mathrm{a}}$
}

\section{Pneumonia and legionellosis mortality in Spain: a time series study}

Background: Pneumonia is a disease with great relevance in public health, as a leading individual cause of infant mortality worldwide. Legionellosis is a respiratory disease with a bacterial origin and two different clinical forms. Aim: To determine pneumonia and legionellosis mortality in Spain. Material and Methods: Time series study of pneumonia and legionellosis in Spain in two periods, from 1997 to 2001 and from 2011 to 2015. Mortality was calculated according to disease and sex, number of deaths and rates per 100,000 inhabitants. Results: Pneumonia mortality in the first period shows a relatively stable and similar tendency according to sex, preferably affecting males. In the second period, pneumonia mortality increased significantly in recent years. Although pneumonia mortality in Spain decreased in both sexes in some age groups (especially $<5$ years), it remained relatively stable in patients aged $>75$ years. Deaths due to legionellosis were relevant in 1997, 1998 and 2000 and increased in the last period. Conclusions: A higher mortality due to pneumonia along the years was identified. Strategies to reduce the incidence and improve the diagnosis of pneumonia, especially in children and older people elderly, are encouraged.

(Rev Med Chile 2021; 149: 433-438)

Key words: Legionellosis; Mortality; Pneumonia; Spain.
${ }^{1}$ Pontificia Universidad Católica del Ecuador. Facultad de Medicina. Quito, Ecuador. aPhD, MPH, MOH \& S, MSC

Trabajo no recibió financiamiento. El autor declara no tener conflictos de interés.

Recibido el 15 de diciembre de 2019, aceptado el 3 de febrero de 2021.

Correspondencia a: Enrique Gea-Izquierdo Pontificia Universidad Católica del Ecuador. Facultad de Medicina. Quito, Ecuador. enriquegea@yahoo.es
L as enfermedades respiratorias tienen un alto impacto a nivel mundial con una gran $\checkmark$ repercusión en términos de mortalidad. La distribución global de esta es heterogénea, inclusive en Europa. Con un promedio de 43,6 muertes por 100.000 habitantes, estas enfermedades fueron la tercera causa de muerte más común en la EU-27, por detrás de las enfermedades cardiovasculares y el cáncer. Dentro del grupo de las enfermedades respiratorias la causa de muerte más frecuente fueron las enfermedades crónicas seguidas de la neumonía ${ }^{1}$. Globalmente la neumonía es un serio problema de salud pública, siendo una de las mayores causas de mortalidad y morbilidad; en particular, respecto a las enfermedades infecciosas, constituye la mayor causa de muerte en el mundo.
A nivel mundial, la enfermedad fue responsable aproximadamente de 2.377.000 muertes en el año 2016. La mortalidad varió según el índice sociodemográfico (SDI), de la octava causa más común en los grupos con alto SDI a la causa más común de muerte en el grupo de naciones de bajo $\mathrm{SDI}^{2}$. En el mundo, la neumonía es una causa frecuente de fallecimiento ${ }^{3}$; tratándose de la sexta causa de muerte general y la causa infecciosa más común de defunción, con una tasa global de 14\%. De hecho, la neumonía es la quinta a novena causa de muerte en los países desarrollados ${ }^{3,4}$. Cabe mencionar que cada año alrededor de $5-10 \%$ de los casos de neumonía mundiales son causados por Legionella, con una tasa de ataque $<5 \%$ y $30 \%$ de tasa de mortalidad si no es tratada la enfermedad. 
De hecho, Legionella spp. ha sido identificada como una causa común de neumonía nosocomial y adquirida en la comunidad.

La neumonía es la principal causa individual de mortalidad infantil en todo el mundo, superando en niños menores de cinco años al total de muertes por el sida, malaria y sarampión ${ }^{5}$. Diversos autores han descrito esta mortalidad en el mundo $0^{6,7}$ a pesar de la dificultad para realizar las estimaciones y las diferencias entre el mundo desarrollado y no desarrollado, calculando que en la actualidad hay más de 2 millones de muertes cada año en niños menores de 5 años ${ }^{8}$. Por lo tanto, la neumonía provoca aproximadamente $19 \%$ de todas las muertes acontecidas en el mundo en niños menores de cinco años ${ }^{6,9}$, estando fuertemente vinculada a la malnutrición, falta de acceso a cuidados sanitarios y pobreza ${ }^{10}$.

En la Unión Europea, la neumonía permanece como la causa más frecuente de muerte por infección, especialmente en los ancianos y aquellos con comorbilidades $^{11-14}$. Aun considerando las bajas tasas globales en Europa, existe una variación significativa en la mortalidad por neumonía entre los países y regiones geográficas ${ }^{15-17}$, con un aumento en la mayoría de los países de la Unión Europea y sustanciales variaciones en las tendencias entre países y $\operatorname{sexos}^{18}$. En España el estudio de las defunciones por neumonía ubica la importancia de este tipo de causa de muerte y su relevancia en comparación con otras enfermedades.

El objetivo del estudio es determinar la mortalidad por neumonía y legionelosis en España para las series 1997-2001 y 2011-2015.

\section{Material y Métodos}

Estudio transversal de series temporales (19972001 y 2011-2015), en pacientes afectados por neumonía o legionelosis en España.

En los criterios de inclusión se estimó el diagnóstico principal de neumonía o legionelosis confirmada. Para la primera se consideró la adquirida en la comunidad y la hospitalaria, y en la segunda adicionalmente EWGLINET. Asimismo, los casos registrados en el Gobierno de España, Ministerio de Ciencia, Innovación y Universidades o equivalente, entre 1997-2001 y 2011-2015. Los criterios de exclusión se aplicaron en pacientes con datos incompletos y en expedientes de pacientes que no cumpliesen con los criterios clínicos y paraclínicos de las enfermedades descritas.

Los datos fueron extraídos del registro, actualizado continuamente, controlado por el CNE (Centro Nacional de Epidemiología) y perteneciente al Instituto de Salud Carlos III, identificando a través del Área de Análisis Epidemiológico y Situación de Salud las cifras de mortalidad y morbilidad. Mediante el uso de estos valores se presentó el estudio de la mortalidad relativa al alcance por neumonía y legionelosis. Los datos tomados de la base ministerial fueron reportados y validados a nivel nacional $y$, por lo tanto, suprime la posibilidad de error de muestreo.

El estudio fue realizado sobre datos secundarios de neumonía (International Classification of Diseases, $9^{\text {th }}$ revision, ICD-9, Chapter VIII: Diseases of the respiratory system, Codes $460-519)^{19} \mathrm{y}$ legionelosis; y series históricas 1997 y 2001, y 20112015. La incidencia anual por 100.000 habitantes fue calculada mediante los datos de la población española para el año correspondiente provenientes del Instituto Nacional de Estadística (INE).

Las variables empleadas fueron: sexo, edad, comunidad autónoma, año, mes, especie (referido a aislamiento de Legionella), serogrupo (referido a aislamiento de Legionella pneumophila), ámbito, categoría de casos y método principal de diagnosis.

\section{Análisis estadístico}

Se determinó la mortalidad por neumonía y sexo, y legionelosis y sexo según las: defunciones y tasas por 100.000 habitantes. El número de defunciones por legionelosis notificados a la red nacional de vigilancia epidemiológica se calculó para los ámbitos comunitario, nosocomial, ambos, EWGLINET, y ámbito comunitario + nosocomial + EWGLINET. Asimismo, se obtuvieron las frecuencias relativas (\%) de la variable cualitativa sexo atendiendo a la mortalidad, y mortalidad promedio y mediana en hombres y mujeres.

La dependencia entre la mortalidad por neumonía y legionelosis en España (ambos sexos) y las series 1997-2001 y 2011-2015 se estableció mediante medidas de asociación. En particular, se determinó la gamma $(\gamma)$, estadístico exacto de Fisher y Kendall-Stuart tau-c $\left(\tau_{c}\right)$. Para ello, se consideraron los años descritos según cada serie y la mortalidad específica para cada enfermedad. Estas últimas fueron agrupadas en intervalos por el número de defunciones y asignación ordinal 
(neumonía [7.000-8.000], [8.001-9.000] y [9.00110.000]; legionelosis [0-10], [11-20] y [21-30]). Asimismo, se calculó el número total de defunciones para ambos sexos, respecto a la neumonía y legionelosis, en cada año de las dos series.

En el cálculo de Fisher se implementó el algoritmo FEXACT $^{20}$. La potencia estadística y el cálculo del tamaño del efecto se obtuvieron a través del programa $\mathrm{G}^{\star}$ Power $^{21}$ Versión 3.1.9.4. Para la gestión de los datos y análisis estadístico se empleó Stata 14.2.

\section{Resultados}

Desde 1992 a 2001 las defunciones se incluyeron en el intervalo aproximado 3.170-4.260, presentando valores menores en la mujer con respecto al hombre. La edad media a la defunción por neumonía fue aproximadamente 76-80 años para los hombres y 82-85 años para las mujeres. En esta serie las defunciones por neumonía, año y sexo se aproximaron bastante, con valores menores en las mujeres. En cambio, en la serie 20102015 las defunciones por neumonía en España sufrieron un ligero incremento, especialmente en el último año. En el sexo femenino los registros fueron menores, pero con un comportamiento similar al de los hombres (Figura 1). En el año 2015 se contabilizó el mayor número de muertes, 5.229 para los hombres y 4.980 en las mujeres, mientras que 2010 supuso la menor cantidad con una frecuencia absoluta prácticamente idéntica en ambos sexos (3.771 en hombres frente a 3.754 en mujeres).

En consonancia con lo descrito en la Figura 1, la Figura 2 muestra la evolución de las tasas por 100.000 habitantes atendiendo al sexo en España
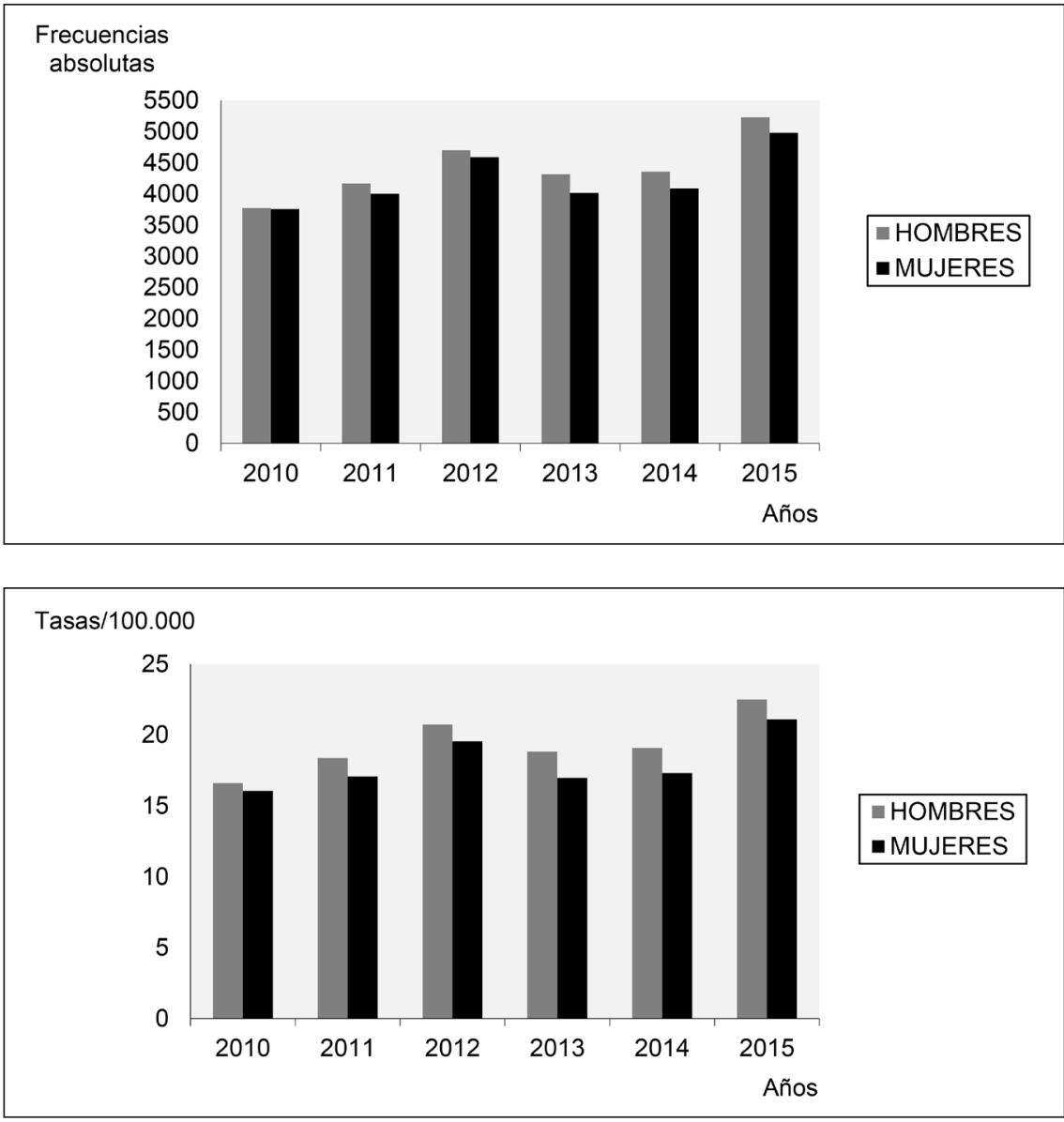

Figura 1. Mortalidad por neumonía y sexo (España, 2010-2015. Número de defunciones).

Figura 2. Mortalidad por neumonía y sexo (España, 2010-2015. Tasas por 100.000 habitantes). 
para los mismos años. En el año 2015 las tasas para los hombres y las mujeres fueron respectivamente 22,50 y 21,09; en comparación con 2010 donde se contabilizaron las más bajas $(16,61$ y 16,06$)$. Los valores representados en las Figuras 1 y 2 indicaron mayores defunciones por neumonía para el sexo masculino aunque sin diferencias notables con el femenino, especialmente en el año 2010. Asimismo, el año en que más defunciones por neumonía se produjeron tanto en hombres como en mujeres fue el 2015. Durante 2011-2015 la distribución de la mortalidad por neumonía en España según el sexo fue de 51,23\% en hombres y $48,77 \%$ en mujeres. Los estadísticos principales de centralización determinados fueron: el promedio (hombres $=4.554$ y mujeres $=4.335$ ) y la mediana (hombres $=4.357$ y mujeres $=4.088$ ).

Respecto a la legionelosis y serie 1997-2002 se observó un mayor número total de defunciones nosocomiales frente a las comunitarias aunque en general la representación de las defunciones por legionelosis en España para la serie y ámbitos descritos mostró una cierta similitud. En EWGLINET el número total de defunciones fue menor que el comunitario y nosocomial. Para los ámbitos comunitario, nosocomial y EWGLINET se identificó el mayor número total de defunciones por año en el 2000, produciéndose para la serie y conjunto de ámbitos 92 defunciones en total.

Entre 2010-2015 el número total de defunciones por legionelosis según el sexo (Figura 3) se manifestó en que en el masculino hubo un muy ligero decremento en la mortalidad; mientras que en el femenino se produjo una alternancia a lo largo de la serie. Respecto a la mortalidad total, fue más alta en el sexo masculino (90) que en el

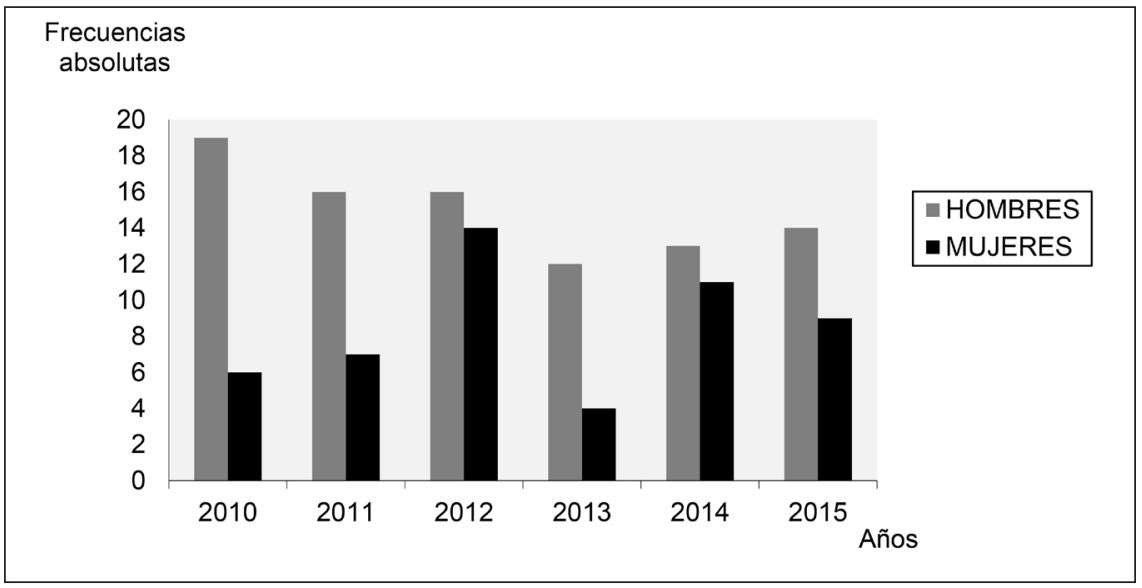

Tasas $/ 100.000$

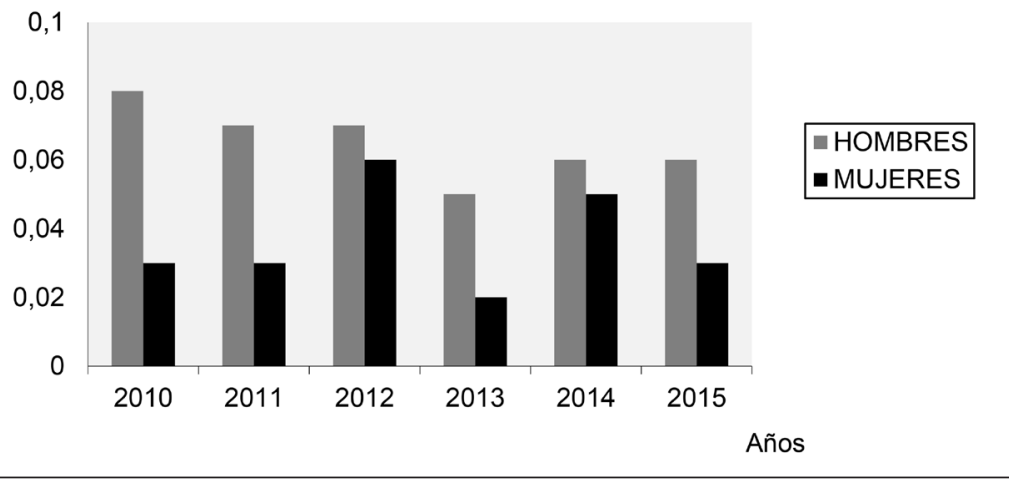

Figura 3. Mortalidad por legionelosis y sexo (España, 2010-2015. Número de defunciones).
Figura 4. Mortalidad por legionelosis y sexo (España, 2010-2015. Tasas por 100.000 habitantes). 
femenino (51). El total de defunciones al año se halló en el intervalo 23-30, excepto en el 2013 que fue 16. Relativo al total, a lo largo de la serie se mantuvo un número aproximado de defunciones.

Lo referente a las tasas por 100.000 habitantes según el sexo (Figura 4) se muestran en la Figura 3. En el hombre se evidenció que los años 2011 y 2012 así como 2014 y 2015 presentaron las mismas, 0,07 y $0,06 \times 100.000$ habitantes, respectivamente. En las mujeres fue de 0,03 en los años 2010, 2011 y 2015. Cabe destacar que en los años 2012 y 2014 las tasas en los dos sexos fueron muy próximas, siendo la mayor en hombres en el año $2010(0,08)$ y la menor en mujeres en el $2013(0,02)$. Los años en que más defunciones por legionelosis se produjeron fueron el 2011 y 2012 en hombres, y en mujeres el último. En la serie 2011-2015, la distribución de la mortalidad por legionelosis en España según el sexo fue de $61,21 \%$ en hombres y $38,79 \%$ en mujeres. Los estadísticos principales de centralización determinados fueron: el promedio (hombres $=14$ y mujeres $=9$ ) y la mediana (hombres $=14$ y mujeres $=9$ ).

Las medidas de asociación calculadas para la mortalidad por neumonía y legionelosis en España, mostraron una $\gamma=1(\mathrm{p}=0,000)$ y un test exacto de Fisher $=0,079$ en la serie 1997-2001; y $\gamma=0,888(\mathrm{p}=0,161)$ y Fisher $=0,206$ en el periodo 2011-2015; mientras que los valores de $\tau_{\mathrm{c}}$ (neumonía) $=0,88(\mathrm{p}=0,000)$ y $\tau_{\mathrm{c}}$ (legionelosis $)=0,64(p=0,007)$. En lo relativo a la relación entre la serie y la mortalidad, partiendo del valor de la $\tau_{c}$ neumonía, se obtuvo el tamaño del efecto $(\mathrm{p}=0,93)$ considerado grande y una potencia estadística de $(1-\beta=0,99)$; mientras que para la legionelosis el tamaño del efecto $(\mathrm{p}=0,80)$ fue de la misma consideración y la potencia estadística $(1-\beta=0,86)$ permitió generalizar los datos.

\section{Discusión}

La vigilancia epidemiológica de la neumonía constituye una herramienta imprescindible en la prevención y control de la enfermedad; y, en particular, en el análisis de las series temporales ${ }^{22}$, ya que permite estudiar y comparar diferentes situaciones. En España, son especialmente relevantes los ámbitos comunitario y nosocomial (neumonía) ${ }^{23}$ y EWGLINET (legionelosis). Atendiendo a las pruebas realizadas, en las dos enfermedades estudiadas se identificó una mayor mortalidad con un aumento de los años. Quizás la legionelosis, que mata aproximadamente 500 personas/año en la Unión Europea, y que tiene una tendencia alcista desde 2005, pudiera acotarse por factores contemplados a determinadas áreas geográficas y ciertos periodos del año. El calentamiento global $\mathrm{y}$ un futuro con consideraciones de temperaturas extremas, induce a predecir un incremento del riesgo de desarrollo del agente biológico ${ }^{24}$. Por lo tanto, parece razonable aumentar el conocimiento mediante la investigación epidemiológica, con nuevas y complementarias variables que puedan servir para contener favorablemente el microorganismo $^{25}$. Especial atención requieren los casos comunitarios, ya que son los mayoritarios, y la identificación de variables contributivas a casos esporádicos. El estudio de las series temporales puede ser considerado como un mecanismo válido en la vigilancia epidemiológica de las enfermedades, ya que permitiría aumentar significativamente la calidad de vida de la población, en su contribución a la promoción, prevención y protección de la salud.

\section{Referencias}

1. European Commission. Eurostat. Estadísticas sobre causas de muerte. Disponible en: http://epp.eurostat. ec.europa.eu/statistics_explained/index.php/Causes_of_death_statistics/es\#Enfermedades_respiratorias [citado el 21 de diciembre de 2017].

2. Naghavi M, Abajobir AA, Abbafati C, Abbas KM, AbdAllah F, Abera SF, et al. Global, regional, and national age-sex specific mortality for 264 causes of death, 19802016: a systematic analysis for the Global Burden of Disease Study 2016. Lancet 2017; 390 (10100): 1151-210.

3. Fassmer AM, Spreckelsen O, Hoffmann F. Incidence of pneumonia in nursing home residents in Germany: results of a claims data analysis. Epidemiol Infect 2018; 146 (9): 1123-9.

4. Guest JF, Morris A. Community-acquired pneumonia: the annual cost to the National Health Service in the UK. Eur Respir J 1997; 10 (7): 1530-4.

5. The United Nations Children's Fund. World Health Organization. Pneumonia: The forgotten killer of children. New York: UNICEF; 2006. Disponible en: http://www. childinfo.org/files/Pneumonia_The_Forgotten_Killer_ of_Children.pdf [citado el 21 de diciembre de 2017].

6. Bryce J, Boschi-Pinto C, Shibuya K, Black RE; WHO 
Child Health and Epidemiology Research Group. WHO estimates of the causes of death in children. Lancet 2005; 365: 1147-52.

7. Williams BG, Gouws E, Boschi-Pinto C, Bryce J, Dye C. Estimates of world-wide distribution of child deaths from acute respiratory infections. Lancet Infect Dis 2002; 2: 25-32.

8. Morris SS, Black RE, Tomaskovic L. Predicting the distribution of under-five deaths by cause in countries without adequate vital registration systems. Int J Epidemiol 2003; 32 (6): 1041-51.

9. Rudan I, Boschi-Pinto C, Biloglav Z, Mulholland K, Campbell H. Epidemiology and etiology of childhood pneumonia. Bull World Health Organ 2008; 86 (5): 408-16.

10. WHO/UNICEF. Global action plan for prevention and control of pneumonia (GAPP). Geneva: WHO; 2009.

11. Jackson ML, Neuzil KM, Thompson WW, Shay DK, Yu $\mathrm{O}$, Hanson CA, et al. The burden of community-acquired pneumonia in seniors: results of a population-based study. Clin Infect Dis 2004; 39 (11): 1642-50.

12. Trotter CL, Stuart JM, George R, Miller E. Increasing hospital admissions for pneumonia, England. Emerg Infect Dis 2008; 14 (5): 727-33.

13. Sligl WI, Majumdar SR. How important is age in defining the prognosis of patients with community-acquired pneumonia? Curr Opin Infect Dis 2011; 24 (2): 142-7.

14. Welte T, Torres A, Nathwani D. Clinical and economic burden of community-acquired pneumonia among adults in Europe. Thorax 2012; 67 (1): 71-9.

15. Gibson GJ, Loddenkemper R, Lundbäck B, Sibille Y. Respiratory health and disease in Europe: the new European lung white book. Eur Respir J 2013; 42 (3): 559-63.

16. Tichopad A, Roberts C, Gembula I, Hajek P, Skoczynska
A, Hryniewicz W, et al. Clinical and economic burden of community-acquired pneumonia among adults in the Czech Republic, Hungary, Poland and Slovakia. PLoS One 2013; 8 (8): e71375.

17. ul Haq R, Rivers P, Umar M. Determinants of differentials in pneumonia mortality in the UK and France. J Health Care Finance 2014; 40 (3): 101-10.

18. Marshall DC, Goodson RJ, Xu Y, Komorowski M, Shalhoub J, Maruthappu M, et al. Trends in mortality from pneumonia in the Europe union: a temporal analysis of the European detailed mortality database between 2001 and 2014. Respir Res 2018; 19 (1): 81.

19. WHO (World Health Organization). International Classification of Diseases, $9^{\text {th }}$ revision, ICD-9. Geneva: Switzerland; 1977.

20. Mehta CR, Patel NR. Algorithm 643 FEXACT: A FORTRAN subroutine for Fisher's exact test on unordered r x c contingency tables. ACM Trans Math Softw 1986; 12: 154-61.

21. Faul F, Erdfelder E, Lang AG, Buchner A. G*Power 3: A flexible statistical power analysis program for the social, behavioral, and biomedical sciences. Behav Res Methods 2007; 39: 175-91.

22. Gea-Izquierdo E. Impacto de la neumonía en España: 1992-2001. Rev Cubana Hig Epidemiol 2014; 52 (3): 346-56.

23. Carratalà J, Garcia-Vidal C. An update on Legionella. Curr Opin Infect Dis. 2010; 23: 152-57.

24. Walker JT. The influence of climate change on waterborne disease and Legionella: a review. Perspect Public Health 2018; 138 (5): 282-86.

25. Gea-Izquierdo E. Water disinfection methods and their affect on legionellosis. Tecnol Cienc Agua 2018; 9 (3): 29-46. 